\title{
Laboratory simulators of the colon microbiome
}

\author{
M.C. Martínez-Cuesta, C. Peláez, T. Requena* \\ Department of Food Biotechnology and Microbiology.Institute of Food Science \\ Research, CIAL (CSIC), Nicolás Cabrera 9, 28049 Madrid, Spain.
}

*Corresponding and senior author: Tel.: +34 91 0017900; E-mail address:

t.requena@csic.es 


\begin{abstract}
Laboratory simulators of the colon are designated to reproduce the composition and functionality of complex microbial communities. The in vitro colonic models range from batch fecal incubations, suitable for metabolic studies, to more complex continuous models, involving one or multiple connected vessels, to mimic different regions of the human colon. Laboratory simulators of the colon microbiome allow the screening of a large number of substances, and are valuable in performing mechanistic studies. Moreover, multistage continuous models are relevant for microbial ecological studies, since they allow long-term experiments, needed to evaluate the spatial and temporal adaptation of the colonic microbiota to dietary ingredients, drugs and toxic or radioactive compounds, without ethical constraints. In vitro models are particularly well suited for screening special functions in the gut of prebiotics or probiotics. The progress made in developing in vitro fermentation models, able to closely mimic the gut microbial environment, can offer remarkable insights into gut microbiota functions or microbial metabolites associated with a disease state, and could be predictive for in vivo situations.
\end{abstract}

Keywords: Colon microbiota; in vitro model; SCFA; diet; prebiotic; probiotic 


\section{Context}

The evaluation of gut microbiota composition and functionality has used mostly fecal samples, which are considered representative of the distal large intestine. Fecal inoculation has also been used in gnotobiotic humanized mouse models, to reproduce the human gut microbiota in vivo ${ }^{1}$.

Laboratory simulators of the colon microbiome are considered excellent tools to allow the screening of a wide range of dietary ingredients, pathogens, drugs, and toxic or radioactive compounds, without ethical constraints. In vitro models vary from batch incubations, using anaerobic conditions and dense fecal microbiota, to more complex continuous models, involving one or multiple connected, $\mathrm{pH}$-controlled reactors inoculated with fecal microbiota, and representing different parts of the human colon ${ }^{2}$.

Laboratory colonic models are inoculated with fecal microbiota, and operated in an anaerobic atmosphere, at temperature and $\mathrm{pH}$ values mimicking physiological conditions. The simulation in vitro of the representative microbial populations from the human gut, encounters the challenges, among others, of reproducing the microbiota complexity, and being representative of a general population. The inoculation prepared from a pool of donors has been suggested to integrate a wider variety of bacterial species, and to produce a standardized and reproducible microbiota. This should lessen the effect of inter-individual variation among donors, for the accurate interpretation of the effects of experimental treatments ${ }^{3}$. In other conditions, such as the exploration of different metabotypes, the colonic models should be inoculated with the fecal microbiome from one individual, to characterize bioactive metabolite-producing phenotypes towards specific compounds, for example polyphenols ${ }^{4}$. 


\section{Key microorganisms and crucial metabolites}

Despite inter-individual diversity, it is possible to identify a human intestinal core microbiota, representing bacteria that are present among all so far analyzed human populations ${ }^{5}$. The bacterial phyla representative of the human gut microbiota are Bacteroidetes, Firmicutes, Actinobacteria, Proteobacteria and Verrucomicrobia. Archaeal representatives consist largely of the methanogens Methanobrevibacter and Methanosphaera. The complexity increases toward the lower taxonomic ranks, from phylum to species level, but analyses of cumulative abundance in metagenomic studies, indicated that bacteria related to Faecalibacterium, Bifidobacterium, Roseburia, Ruminococcus, Subdoligranulum, Prevotella, Ruminococcus, Akkermansia and Oscillospira, represent common core bacteria in the Western adult population ${ }^{6}$.

Additionally, the intestinal minimal functional core contains specific pathways involved in resistance to bile, ability to grow in an anaerobic environment, and the metabolic capabilities towards degradation of a wide range of polysaccharides, and utilization of available amino acids and lipids.

The major metabolic products of anaerobic fermentation by gut microbial communities that colonize the mammalian gut are short chain fatty acids (SCFA), mainly acetate, propionate and butyrate. The release of these microbial metabolites causes decrease of the luminal $\mathrm{pH}$, which reduces the risk of pathogen colonization, and favors absorption of some nutrients. SCFA are also absorbed efficiently by the gut mucosa, and have important impacts upon human physiology ${ }^{7}$.

Butyrate is used preferentially as an energy source by colonocytes, and has demonstrated anti-inflammatory activity and protective effects against colorectal cancer. Propionate promotes satiety and reduces cholesterol ${ }^{8}$. In addition, butyrate and 
propionate have also been reported to induce the differentiation of T-regulatory cells, assisting the control of inflammation. Members of the Clostridium clusters IV and XIVa are exceptional inducers of T-regulatory cells in the colon, and are considered as therapeutic options for intestinal inflammatory diseases and allergies ${ }^{9}$.

Intestinal microbiota also produces some biogenic compounds such as free vitamins, bioactive peptides, and neurotransmitters like $\gamma$-amino butyric acid (GABA). The intestinal microbiota participates in the detoxification of xenobiotics and contaminants, and the biotransformation of drugs or their metabolites ${ }^{10}$.

\section{General models of the colon microbiome}

\section{Batch fermentation models}

Batch models are used for short-term fermentation studies, in which specific strains or fecal microbial communities are maintained under anaerobic conditions. These models range from closed bottles, used to grow defined (single or mixed) bacterial strains, to controlled reactors inoculated with fecal microbiota suspensions. These batch fermentations are of particular interest for initial screening assays of microbial metabolism, due to the high-inter individual variations in microbiota composition, as well as to test different sources or doses of compounds. The model is also useful to elucidate microbial metabolism pathways, although it is limited by substrate depletion, and accumulation of end products (Table 1).

\footnotetext{
$* * *$ Insert Table $1 * * *$
}

\section{Dynamic fermentation models}


Single or multistage continuous models are relevant for microbial ecological studies, since they allow long-term experiments, needed to evaluate the spatial and temporal adaptation of the colonic microbiota to dietary compounds (Table 1).

Continuous single-stage fermentation models are often designed to simulate the proximal colon conditions, and to reproduce its metabolic activity. The computercontrolled, dynamic in vitro proximal colon model (TIM-2) was developed by the TNO in the Netherlands ${ }^{11}$. This model has unique features, as it includes host functions such as peristaltic mixing, and water and metabolite absorption. The TIM-2 model consists of four interconnected glass compartments, with a flexible membrane inside, and water (37 $\left.{ }^{\circ} \mathrm{C}\right)$ between the glass jacket and the membrane.

Peristaltic movements are achieved by applying pressure on the water at regular intervals, causing contraction of the flexible membrane, and then peristaltic waves, which mix the luminal content and move it through the system. The TIM-2 contains dialysis membranes (molecular mass cut of $50 \mathrm{kDa}$ ), in the lumen of the reactor, that prevent accumulation of microbial metabolites. The model is equipped with a level sensor to maintain the colon simulator volume at $120 \mathrm{~mL}$, and $\mathrm{pH}$ control at 5.8.

An in vitro proximal colon model, composed of a single-stage culture, that uses several vessels to allow testing in parallel the effects of different treatments, on the same complex gut microbiota (biological replication), was developed at ETH in Zurich, Switzerland $^{12}$. The model PolyFermS uses immobilized fecal microbiota in a first-stage inoculum reactor, representative of the complex bacterial community, which is present both at planktonic and sessile (biofilm-associated) states in the colon. Thus, the fecal inoculum is immobilized in 1-2 $\mathrm{mm}$ diameter gel beads, on a dispersion process in a two-phase system, composed of gellan and xanthan gums. The first-stage inoculum 
reactor, seeded with immobilized fecal microbiota, is used to continuously inoculate a set of parallel second-stage reactors.

Dynamic multistage-fermentation models are mostly based on the Reading model, first described by Gibson et al. ${ }^{13}$.The model consists of three vessels of increasing volume size connected in series, replicating the proximal (V1; $0.3 \mathrm{~L})$, transverse (V2; $0.5 \mathrm{~L})$ and distal (V3; $0.8 \mathrm{~L})$ colon regions. The operating conditions include setting of $\mathrm{pH}$ for the three vessels at 5.5, 6.2 and 6.8, respectively, and a total retention time of 62.7 h. Each fermenter is magnetically stirred, and maintained under an atmosphere of $\mathrm{CO}_{2}$.

The authors also developed a nutritious medium, which has been extensively used in both batch and dynamic fermentation models. The medium consists of protein substrates (casein and peptone), complex carbohydrates (pectin, xylan, arabinogalactan and resistant starches), that are not digested by gastrointestinal enzymes, and a mixture of bile salts, minerals and vitamins. Vessel V1 is fed with the medium from a reservoir (R1), and can also receive mucin from reservoir R2. The model can operate at different retention times, to assess the effect of colonic transit on carbohydrate utilization, metabolite formation, and the ecology of numerically important bacterial populations.

A further development of the Reading model is the Simulator of the Human Intestinal Microbial Ecosystem (SHIME) (Ghent University, Belgium). This system comprises a series of five double-jacketed glass reactors, that simulates the stomach and small intestine (mimicking the ingestion and digestion processes), connected to the three-stage continuous colonic model through peristaltic pumps ${ }^{14}$. The system operates semi-continuously, with intermittent supplementation of nutritional medium, and the removal of microbial suspension. The first two reactors follow a fill-and-draw principle, adding three times a day a defined nutritional medium to the gastric compartment, and 
pancreatic and bile liquid to the small intestine compartment. Upon digestion in the gastric and intestine compartments, the slurry is pumped in the ascending colon vessel, where colon fermentation is initiated. Retention colon time is primarily modulated through a change in compartment volume and, depending on the human target group of interest, may vary from $24 \mathrm{~h}$ to $72 \mathrm{~h}$.

\section{Automated simulators of the colon microbiome: The SIMGI and BFBL models}

We developed the SIMGI (SIMulator Gastro-Intestinal) model at the Institute of Food Science Research CIAL (Madrid, Spain), as a fully automated gastrointestinal multi-chamber simulator, to dynamically reproduce the physiological processes taking place during digestion in the stomach and small intestine, as well as to simulate the colonic microbiota, responsible for metabolic bioconversions in the large intestine $e^{15}$.

The gastric compartment consists of two cylindrical transparent and rigid methacrylate plastic modules, covering a reservoir of flexible silicone walls, where the gastric content is mixed by peristaltic movements. The gastric peristalsis is achieved by the pressure of thermostated water, that flows in the jacket between the plastic modules and the reservoir, and keeps the temperature of the gastric content at $37^{\circ} \mathrm{C}$. The meal received by the stomach compartment is mixed with gastric electrolytes and enzymes, and the decrease of $\mathrm{pH}$ is automatically-controlled to follow the curve resulting from a linear fit of data, representing experimental in vivo conditions.

The stomach emptying is programmable, to allow the modification of the emptying curve shape, depending on liquid, semisolid or solid foods. The process of digestion of whey proteins in this compartment has recently been demonstrated to represent in vivo data, with a stepped appearance of peptides from serum albumin and $\alpha$ - 
lactalbumin, and the partial passage of undegraded $\beta$-lactoglobulin to the upper intestine $^{16}$.

The small intestine consists in a double jacket glass reactor vessel, continuously stirred $(150 \mathrm{rpm})$, that receives the gastric content, which is then mixed with pancreatic juice and bile salts. The intestinal content is digested during $2 \mathrm{~h}$ at $37^{\circ} \mathrm{C}$, and kept at neutral $\mathrm{pH}$ values. The digestion temperature is regulated by pumping thermostated water into the space between the glass jackets, and nitrogen is continuously flushed into the medium to induce anaerobic conditions during digestion. After the small intestinal digestion, the whole content of the vessel is automatically transferred, at a flow rate of 5 $\mathrm{mL} / \mathrm{min}$, to the proximal colon compartment.

The stages of the large intestine are simulated in three double jacket-glass continuous reactors, which are interconnected by pipes and peristaltic valve pumps. The $\mathrm{pH}$ in the colonic units, named ascending $\mathrm{AC}$, transverse $\mathrm{TC}$ and descending $\mathrm{DC}$ colon, is controlled to 5.6 in the AC, 6.3 in the TC and 6.8 in the DC compartments. When the digested content of the small intestine is transferred to the proximal colon vessel (AC), the transit of colonic content between the AC, TC and DC compartments is simultaneously initiated at the same flow rate. Reactors are continuously purged with nitrogen, creating a bubbling effect in the colonic content, to induce anaerobic conditions for the oxygen-sensitive intestinal microbial communities.

The SIMGI design is aimed to dynamically operate with the five units simulating the whole gastrointestinal process, and constitute a CIAL Institute Platform for digestion and fermentation services. Based on it, we have proceeded with the development of a specific colon simulator, adapted to laboratory-scale conditions, named Simulator BFBL (after Spanish words for Functional Biology of Lactic acid Bacteria; Fig. 1). The BFBL-laboratory simulator of the colon microbiome is also a 
three-stage fermentation model, with the direct feeding of the small intestine with nutritious medium. After digestion, the controlled transit of the small intestinal content to the AC, DC and TC vessels is activated, for reproducing the colon region-specific microbiota.

The system is fully automated, and placed in a biological safety cabinet, that allows therapeutical protocols involving food-borne pathogens. In addition, the cabinet air flow prevents environmental contamination with microaerophilic opportunists. An additional upgrade in the BFBL simulator is the control of reactor volumes with level sensors, that activate transport of the colonic content, to guarantee the desired colon retention times. In addition, nitrogen is flushed to remove oxygen from the head space, only during digestion and fluid transit between compartments, and without the bubbling effect. This would circumvent a direct nitrogen influence on the bacterial metabolism and fermentation yields, mainly regarding hydrogenotrophic colon organisms such as acetogens, sulfate-reducing bacteria and methanogens ${ }^{17}$.

The BFBL simulator stores the input monitored values from the temperature and $\mathrm{pH}$ sensors. The automated representation of $\mathrm{pH}$ curves and pumping of acid and base to the compartments, are indicative of microbial development and metabolism. This information is crucial as control of the process, since the analyses of metabolites and fermentation products are delayed, and not measured online.

The laboratory-scale setting of the BFBL simulator facilitates its flexiblemodulating characteristics to operate with individual reactors. Additionally, it can incorporate devices simulating the gut microbiota-host interactions. Assays for evaluating this type of valuable interaction are currently approached by co-culturing colon-region specific microbiota suspensions, from the AC, TC and DC vessels, with epithelial or immune cells. The simulation of bacterial adhesion and intestinal 
absorption of microbial metabolites is planned with the setting up of microbial/mucosa interfaces, in the lumen of the colon reactors, and by including dialysis devices between compartments.

***Insert Figure 1***

\section{Protocols and applications}

The three-stage colon simulators aligned in series imply that a sequential feeding of growth medium occurs. Thus, the first vessel has a high availability of substrate, representing a rapid bacterial growth rate, and is operated at an acidic $\mathrm{pH}$, similar to events in the proximal colon. In contrast, the final vessel resembles the neutral $\mathrm{pH}$, slow bacterial proliferation rate, and low substrate availability, which is characteristic of more distal regions. The viability of the in vitro gut microbiota is dependent on the continuous replenishment of nutrients and the control of physiological temperature and anaerobic conditions.

The control of these factors allows the establishment of steady-state conditions, with respect to both microbial composition and metabolic activity, whereas the control of defined $\mathrm{pH}$ values, downstream nutrient limitations, and retention times in the different vessels, allows a region-specific differentiation of the colonic microbiota in terms of metabolic activity and microbial communities.

The development of a colon region-specific microbial ecosystem occurs after the stabilization period in the simulator, and it is needed before starting any experimental approach $^{15}$. Differences observed between the compartments after stabilization indicate that Bacteroides are more representative in the $\mathrm{AC}$ and $\mathrm{TC}$ compartments than in the DC reactor, whereas the butyrate-producing groups Clostridium leptum and 
Ruminoccocus (cluster IV), are less represented in the proximal colon compartment (AC) than in the distal vessels (TC and DC).

Other butyrate-producing bacteria such as Blautia coccoides-Eubacterium rectale group (cluster XIVa), remain equivalent in all colonic compartments (8.1-8.6 log copy number $/ \mathrm{mL}$ at the end of the stabilization period). The carbohydrate-rich conditions of the proximal vessel (AC) favor the predominance of Bacteroides, which are characterized by a marked ability to utilize a wide variety of polysaccharides ${ }^{18}$. Interestingly, when the stabilization period was adapted to simulate an obese-associated microbiota, by using an overweight donor and high energy-content nutritive medium (increased content of high-glycaemic index carbohydrates, such as digestible starch and fructose), the microbial community profiles of the three colon compartments clustered together independently of the carbohydrate content of the diets ${ }^{19}$. The study showed that, in average, the distal colon regions were enriched with Bifidobacterium, C. leptum, Ruminococcus, Akkermansia, Faecalibacterium and Roseburia.

The stabilization is also required to provide a steady-state environment, where the metabolism of the microbial community can be evaluated during long-term experimental dietary interventions. Evaluation of microbial metabolism is usually addressed by measuring short chain fatty acids (SCFA) and ammonium contents. Overall, the net SCFA production, up to functional stability, is highest in the AC compartment, in correspondence with the carbohydrate-excess conditions, although total content accumulates in the distal compartments, due to the lack of absorption steps.

Acetate, propionate and butyrate are the main metabolites measured in colon simulators and, therefore, are representative of in vivo conditions. Formate, succinate and lactate can also be detected, although they are almost only produced in the AC compartment. Succinate and lactate can be further metabolized within the colon, and 
turned into propionate and butyrate, respectively, through cross-feeding by gut bacteria $^{8}$. Ammonium is also evaluated over time, and is considered representative of proteolytic microbial metabolism.

Although proteolysis occurs throughout the entire colon model, average values increase along the colon reactors. Depending on the conditions assayed (diet interventions, development of microbial communities representative of dysbiosis associated to intestinal pathologies, food ingredients, etc.), specialized analytical methodologies targeting the formation of specific intermediate and end metabolites are evaluated ${ }^{4}$.

\section{Therapeutical and other strategical applications}

The dynamic simulators have been developed with the purpose to establish in vitro a relatively stable microbial ecosystem under physiologically relevant colon conditions. The models mimic physiological parameters, based in temperature, anaerobiosis, $\mathrm{pH}$ and retention times, emulating measurements made in in vivo conditions. Due to this, these validated systems are frequently used to test experimental substrates, and could be predictive for in vivo situations ${ }^{2}$.

In vitro models are particularly well suited for screening prebiotics or probiotics for special functions in the gut, before moving to in vivo investigation. A comparison of TIM-2 and SHIME, to study long-chain arabinoxylans as potential prebiotics, has demonstrated that both models similarly revealed a compound-specific modulation of prebiotics, in terms of SCFA production, and stimulation of specific Bifidobacterium species $^{20}$. Since the majority of carbohydrate breakdown occurs in the proximal colon region, dynamic multistage-fermentation models are also valuable for identifying slowly fermentable prebiotics. 
Viability of probiotics can be analyzed during daily feeding of the laboratory simulators, and after ending dietary interventions, in order to evaluate competitive and colonization advantages. The incorporating of mucin-covered surfaces in the colon simulators, e.g. M-SHIME, allows differentiation of probiotic adhesion capabilities to the mucosal environment, thereby elucidating their potential role in human health ${ }^{21}$.

The suitability of the SIMGI and BFBL laboratory automated simulators of the colon microbiome to reproduce human conditions associated to changes in dietary lifestyles, has been evaluated by the microbiological and metabolic changes of an obese-associated colonic microbiota, after the supplementation of the nutritive medium with oligosaccharides derived from lactulose. The comparison of SCFA and ammonium formation, during the microbiota stabilization period (high energy diet), and the substitution of easily digestible carbohydrates by the oligosaccharides derived from lactulose, indicated that the prebiotic allowed the development of fermentative functionality, maintaining the net production of butyrate, with potential beneficial effects on health, and avoiding a full transition to proteolytic metabolism profiles ${ }^{19}$.

In addition, we have observed differences in total SCFA and ammonium production between overweight and lean microbiota donor samples (Fig. 2). There are reports describing an intestinal obesogenic microbiome signature, characterized by a higher capacity of energy harvest, that persists after successful dieting, and contributes to faster weight regain and metabolic aberrations, commonly referred to as the "yo-yo effect $^{22}$. The results we have observed in vitro indicate that the higher metabolism of the obesogenic microbiota seems to occur in the transverse and distal colon regions (Fig. 2).

$* * *$ Insert Figure 2***

\section{Concluding remarks}


The developing of in vitro fermentation models, able to closely mimic the gut microbial environment, can offer remarkable insights into gut microbiota diversity and key functions required for healthy intestinal homeostasis. However, the laboratory in vitro colon simulators are still unsuccessful in the analysis of the mutual communication between the gut bacteria and the intestinal epithelium, or in the simulation of disease conditions of the host. Accordingly, in vitro findings should be fully validated in human studies. 


\section{References}

1. Martin R, Bermudez-Humaran LG, Langella P. Gnotobiotic rodents: An in vivo model for the study of microbe-microbe interactions. Frontiers in Microbiology 2016; 7:409.

2. Aguirre $M$, Venema $K$. Challenges in simulating the human gut for understanding the role of the microbiota in obesity. Beneficial Microbes 2017; 8(1): 31-53.

3. O'Donnell MM, Rea MC, O'Sullivan O, Flynn C, Jones B, McQuaid A, Shanahan F, Ross RP. Preparation of a standardised faecal slurry for ex-vivo microbiota studies which reduces inter-individual donor bias. Journal of Microbiological Methods 2016; 129: 109-16.

4. Barroso E, Van de Wiele T, Jimenez-Giron A, Munoz-Gonzalez I, Martin-Alvarez PJ, Moreno-Arribas MV, Bartolome B, Pelaez C, Martinez-Cuesta MC, Requena T. Lactobacillus plantarum IFPL935 impacts colonic metabolism in a simulator of the human gut microbiota during feeding with red wine polyphenols. Applied Microbiology and Biotechnology2014; 98(15): 6805-15.

5. Mancabelli L, Milani C, Lugli GA, Turroni F, Ferrario C, van Sinderen D, Ventura M. Meta-analysis of the human gut microbiome from urbanized and pre-agricultural populations. Environmental Microbiology 2017; 19(4): 1379-90.

6. Shetty SA, Hugenholtz F, Lahti L, Smidt H, de Vos WM. Intestinal microbiome landscaping: insight in community assemblage and implications for microbial modulation strategies. FEMS Microbiology Reviews2017; 41(2): 182-99.

7. Rios-Covian D, Ruas-Madiedo P, Margolles A, Gueimonde M, de Los Reyes-Gavilan CG, Salazar N. Intestinal short chain fatty acids and their link with diet and human health. Frontiers in Microbiology 2016; 7: 185.

8. Louis P, Flint HJ. Formation of propionate and butyrate by the human colonic microbiota. Environmental Microbiology2017; 19(1): 29-41.

9. El Hage R, Hernandez-Sanabria E, Van de Wiele T. Emerging trends in "smart probiotics": Functional consideration for the development of novel health and industrial applications. Frontiers in Microbiology 2017; 8: 1889.

10. Spanogiannopoulos P, Bess EN, Carmody RN, Turnbaugh PJ. The microbial pharmacists within us: A metagenomic view of xenobiotic metabolism. Nature Reviews in Microbiology 2016; 14(5): 273-87.

11. Minekus M, Smeets-Peeters M, Bernalier A, Marol-Bonnin S, Havenaar R, Marteau P, Alric M, Fonty G, Huis in't Veld JH. A computer-controlled system to simulate conditions of the large intestine with peristaltic mixing, water absorption and absorption of fermentation products. Applied Microbiology and Biotechnology 1999; 53(1): 108-14.

12. Zihler Berner A, Fuentes S, Dostal A, Payne AN, Vazquez Gutierrez P, Chassard C, Grattepanche F, de Vos WM, Lacroix C. Novel Polyfermentor intestinal model (PolyFermS) for controlled ecological studies: validation and effect of pH. PloS one 2013; 8(10): e77772. 13. Gibson GR, Cummings JH, Macfarlane GT. Use of a three-stage continuous culture system to study the effect of mucin on dissimilatory sulfate reduction and methanogenesis by mixed populations of human gut bacteria. Applied and Environmental Microbiology1988; 54(11): 2750-5. 
14. Molly K, Vande Woestyne M, Verstraete W. Development of a 5-step multi-chamber reactor as a simulation of the human intestinal microbial ecosystem. Applied Microbiology and Biotechnology1993; 39(2): 254-8.

15. Barroso E, Cueva C, Pelaez C, Martinez-Cuesta MC, Requena T. Development of human colonic microbiota in the computer-controlled dynamic SIMulator of the Gastrolntestinal tract SIMGI. LWT-Food Science and Technology 2015; 61(2): 283-9.

16. Miralles B, Del Barrio R, Cueva C, Recio I, Amigo L. Dynamic gastric digestion of a commercial whey protein concentratedagger. Journal of the Science of Food and Agriculture2017.

17. Feria-Gervasio D, Tottey W, Gaci N, Alric M, Cardot JM, Peyret P, Martin JF, Pujos E, Sebedio JL, Brugere JF. Three-stage continuous culture system with a self-generated anaerobia to study the regionalized metabolism of the human gut microbiota. Journal of Microbiological Methods2014; 96: 111-8.

18. Wexler AG, Goodman AL. An insider's perspective: Bacteroides as a window into the microbiome. Nature Microbiology2017; 2: 17026.

19. Barroso E, Montilla A, Corzo N, Pelaez C, Martinez-Cuesta MC, Requena T. Effect of lactulose-derived oligosaccharides on intestinal microbiota during the shift between media with different energy contents. Food Research International2016; 89(Pt 1): 302-8.

20. Van den Abbeele P, Venema K, Van de Wiele T, Verstraete W, Possemiers S. Different human gut models reveal the distinct fermentation patterns of arabinoxylan versus inulin. Journal of Agricultural and Food Chemistry2013; 61(41): 9819-27.

21. Van den Abbeele P, Marzorati M, Derde M, De Weirdt R, Joan V, Possemiers S, Van de Wiele T. Arabinoxylans, inulin and Lactobacillus reuteri 1063 repress the adherent-invasive Escherichia coli from mucus in a mucosa-comprising gut model. NPJ Biofilms and Microbiomes2016; 2: 16016.

22. Thaiss CA, Itav S, Rothschild D, MeijerMT, Levy M, Moresi C, Dohnalova L, Braverman S, Rozin S, Malitsky S, Dori-Bachash M, Kuperman Y, Biton I, Gertler A, Harmelin A, Shapiro H, Halpern Z, Aharoni A, Segal E, Elinav E. Persistent microbiome alterations modulate the rate of post-dieting weight regain. Nature 2016; 540: 544-51. 
Table 1. Advantages and limitations of in vitro laboratory simulators of the colon microbiome

\begin{tabular}{|c|c|c|}
\hline Model & Advantages & Limitations \\
\hline Batch & $\begin{array}{l}\text { Easy to operate. Screening studies and inter-individual variability. } \\
\text { Matrix/doses and metabolism assessment. Cost-effective. }\end{array}$ & $\begin{array}{l}\text { Short-term studies. Substrate depletion and } \\
\text { metabolite accumulation to inhibitory levels. }\end{array}$ \\
\hline Dynamic & Control of environmental parameters. Continuous replenishment. & No host functionality, and short-time studies. \\
\hline Immobilized & $\begin{array}{l}\text { High-cell density and long-term studies of a continuous } \\
\text { fermentation system, with immobilized fecal microbiota. }\end{array}$ & $\begin{array}{l}\text { No simulation of water and metabolite absorption. } \\
\text { Home-designed and expensive. }\end{array}$ \\
\hline Multistage & $\begin{array}{l}\text { Colon-region specific research. Microbiome stability over a } \\
\text { long timeframe. Multi-parametric control. }\end{array}$ & $\begin{array}{l}\text { No host functionality. } \\
\text { Home-designed and expensive }\end{array}$ \\
\hline $\begin{array}{l}\text { Host interaction } \\
\text { devices }\end{array}$ & $\begin{array}{l}\text { TIM-2, metabolite absorption and peristalsis } \\
\text { M-SHIME, intestinal mucus simulation }\end{array}$ & $\begin{array}{l}\text { No host immuno-endocrine response.Expensive. } \\
\text { No feed-back mechanisms or host immuno-endocrine } \\
\text { response. Expensive. }\end{array}$ \\
\hline
\end{tabular}


Fig. 1. BFBL-laboratory simulator of the colon microbiome

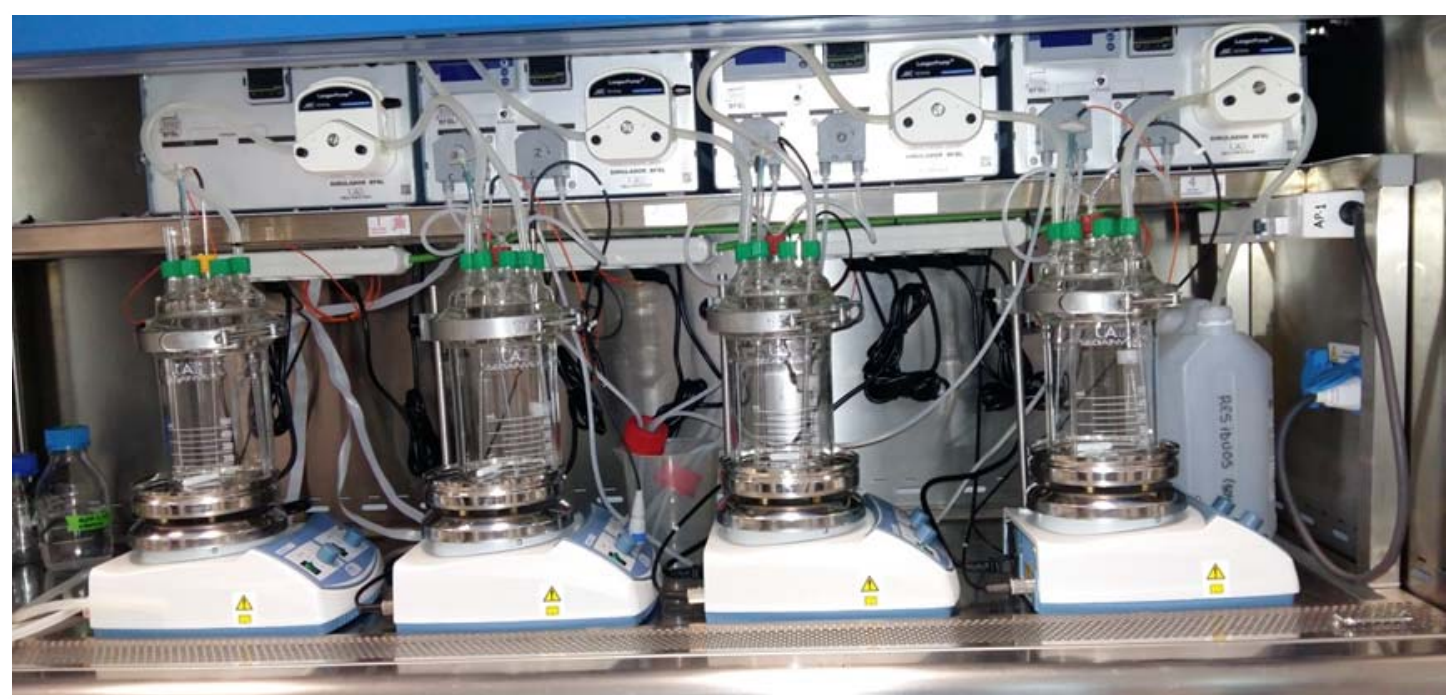


Fig. 2.Total SCFA (A) and ammonium (B) production (mM) by the microbiome from overweight (OW) and lean (L) donors, in the ascending (AC), transverse (TC) and descending colon (DC) reactors of the BFBL-laboratory simulator.

A

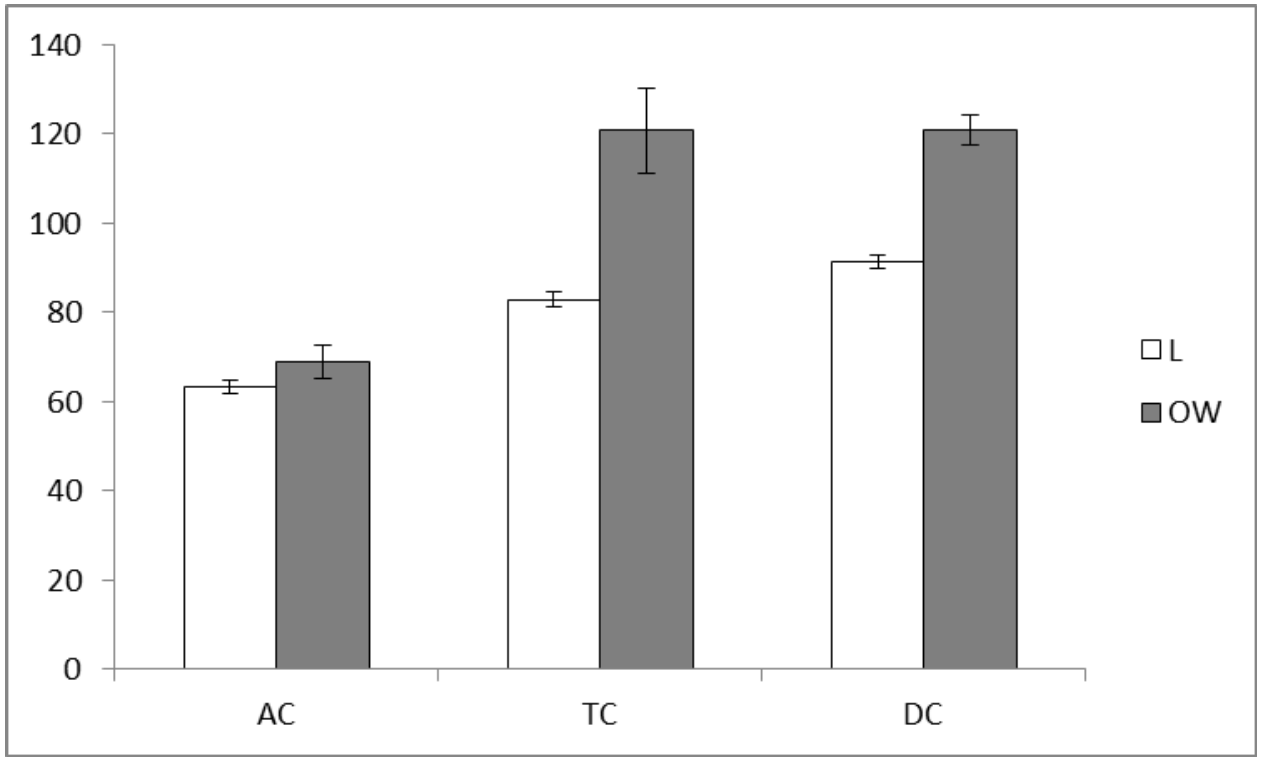

B

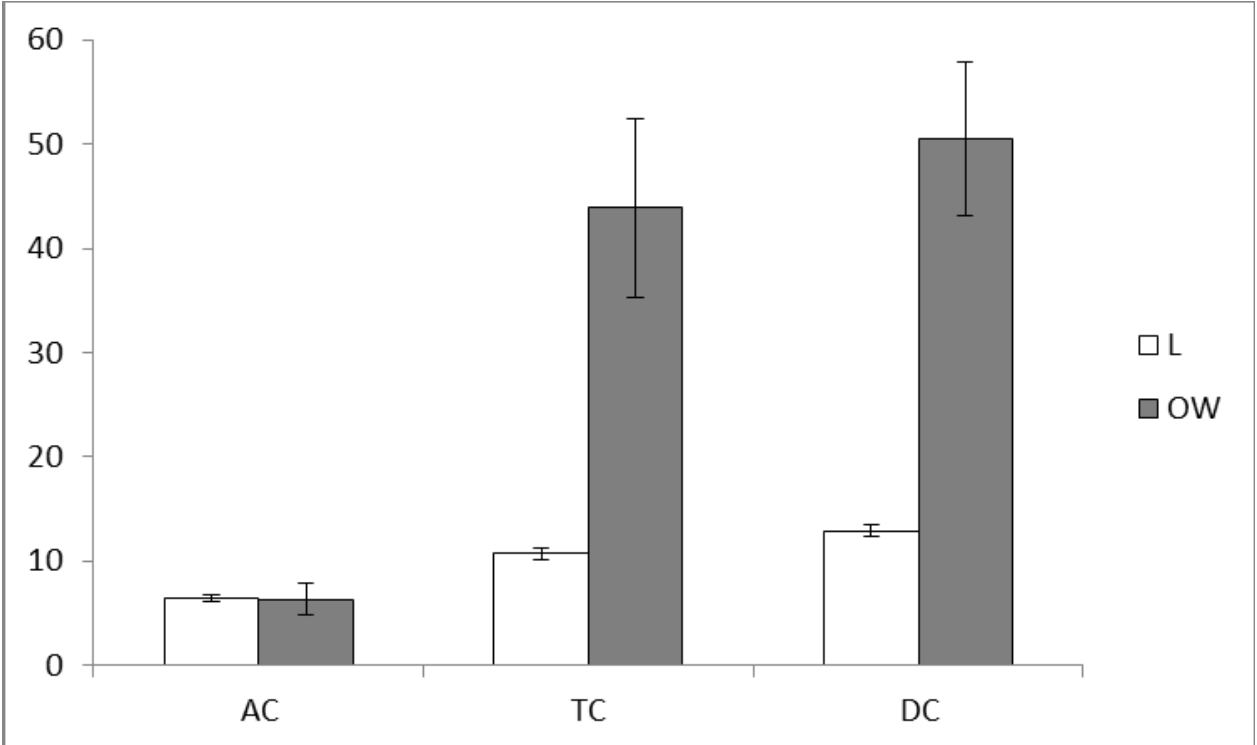

